\title{
On Parallelism of Half-Lightlike Submanifolds of Indefinite Kenmotsu Manifolds
}

\author{
Wenjie Wang ${ }^{1}$ and Ximin Liu ${ }^{2}$ \\ ${ }^{1}$ Department of Mathematics, South China University of Technology, Guangzhou, Guangdong 510641, China \\ ${ }^{2}$ School of Mathematical Sciences, Dalian University of Technology, Dalian, Liaoning 116024, China
}

Correspondence should be addressed to Ximin Liu; ximinliu@dlut.edu.cn

Received 5 April 2013; Accepted 13 May 2013

Academic Editor: Reza Saadati

Copyright (c) $2013 \mathrm{~W}$. Wang and X. Liu. This is an open access article distributed under the Creative Commons Attribution License, which permits unrestricted use, distribution, and reproduction in any medium, provided the original work is properly cited.

We mainly investigate the parallelism of half-lightlike submanifolds of indefinite Kenmotsu manifolds. It is proved that a tangential half-lightlike submanifold $M$ of an indefinite Kenmotsu space form $(\bar{M}(c), \bar{g})$ with semiparallel second fundamental form $h$ either satisfies $c=-1$ or is $(\bar{J}(\operatorname{Rad}(T M)), T M)$-mixed geodesic.

\section{Introduction}

As the intersection of normal bundle and tangent bundle of a submanifold of a semi-Riemannian manifold may not be trivial, it is more difficult and interesting to study the geometry of lightlike submanifolds than nondegenerate submanifolds. The two standard methods to deal with the above difficulties were developed by Kupeli [1], Duggal and Bejancu [2], Duggal and Jin [3], and Duggal and Sahin [4], respectively. Let $M$ be a lightlike submanifold immersed in a semi-Riemannian manifold, it is obvious to see that there are two cases of codimension 2 lightlike submanifolds, since for this type the dimension of their radical distributions is either 1 or 2 . A codimension 2 lightlike submanifold $M$ of a semi-Riemannian manifold $\bar{M}$ is called a half-lightlike submanifold [5] if $\operatorname{dim}(\operatorname{Rad}(T M))=1$, where $\operatorname{Rad}(T M)$ denotes the degenerate radical distribution of $M$. For more results about halflightlike submanifolds, we refer the reader to $[4,6,7]$.

In the theory of submanifolds of Riemannian manifolds, the parallel and semiparallel immersions were studied by Ferus [8] and Deprez [9], respectively. Recently, Massamba [10-13] and Upadhyay and Gupta [14] studied the parallel and semiparallel lightlike hypersurfaces of an indefinite Sasakian, Kenmotsu, and cosymplectic manifolds, respectively. However, the parallel and semiparallel half-lightlike submanifolds of an indefinite Kenmotsu manifolds have not yet been considered. The aim of this paper is to investigate the parallelism of half-lightlike submanifolds of indefinite Kenmotsu manifolds.
This paper is organized in the following way. In Section 2, we provide some well-known basic formulas and properties of indefinite Kenmotsu manifolds and half-lightlike submanifolds. Section 3 is devoted to presenting some main results on semiparallel half-lightlike submanifolds of indefinite Kenmotsu space form. Finally, in Section 4, some properties of parallel half-lightlike submanifolds of indefinite Kenmotsu manifolds are investigated.

\section{Preliminaries}

In this section, we follow Duggal and Sahin [4] for the notation and fundamental equations for half-lightlike submanifolds of indefinite Kenmotsu manifolds. A $(2 n+1)$-dimensional semi-Riemannian $(\bar{M}, \bar{g})$ is said to be an indefinite Kenmotsu manifold if it admits a normal almost contact metric structure $(\bar{J}, \zeta, \theta, \bar{g})$, where $\bar{J}$ is a tensor field of type $(1,1), \zeta$ is a vector field which is called characteristic vector field, $\theta$ is a 1 -form, and $\bar{g}$ is the semi-Riemannian metric on $\bar{M}$ such that

$$
\begin{gathered}
\bar{J}^{2} X=-X+\theta(X) \zeta, \quad \bar{J} \zeta=0, \quad \theta \circ \bar{J}=0, \quad \theta(\zeta)=1 \\
\theta(X)=\bar{g}(\zeta, X), \quad \bar{g}(\bar{J} X, \bar{J} Y)=\bar{g}(X, Y)-\theta(X) \theta(Y) \\
\left(\bar{\nabla}_{X} \bar{J}\right) Y=-\bar{g}(\bar{J} X, Y) \zeta+\theta(Y) \bar{J} X \\
\bar{\nabla}_{X} \zeta=-X+\theta(X) \zeta
\end{gathered}
$$


for any $X, Y \in \Gamma(T \bar{M})$, where $\bar{\nabla}$ denotes the Levi-Civita connection of a semi-Riemannian metric $\bar{g}$.

A plane section of an indefinite Kenmotsu manifold $(\bar{M}, \bar{J}, \zeta, \theta, \bar{g})$ is called a $\bar{J}$-section if it is spanned by a unit vector field $X$ orthogonal to $\zeta$ and $\bar{J} X$, where $X$ is a nonnull vector field on $\bar{M}$. The sectional curvature $K(X, \bar{J} X)$ of a $\bar{J}$ section is called a $\bar{J}$-sectional curvature. If $\bar{M}$ has a constant $\bar{J}$ sectional curvature $c$ which does not depend on the $\bar{J}$-section at each point, then $c$ is a constant and $\bar{M}$ is called a Kenmotsu space form, denoted by $(\bar{M}(c), \bar{g})$. The curvature tensor $\bar{R}$ of an indefinite Kenmotsu space form $(\bar{M}(c), \bar{g})$ is given in [4] as follows:

$$
\begin{aligned}
& \bar{R}(X, Y) Z \\
&=\frac{c-3}{4}\{\bar{g}(Y, Z) X-\bar{g}(X, Z) Y\} \\
&+\frac{c+1}{4} \\
& \times\{\theta(X) \theta(Z) Y-\theta(Y) \theta(Z) X \\
&+\bar{g}(X, Z) \theta(Y) \zeta-\bar{g}(Y, Z) \theta(X) \zeta \\
&+\bar{g}(\bar{J} Y, Z) \bar{J} X-\bar{g}(\bar{J} X, Z) \bar{J} Y-2 \bar{g}(\bar{J} X, Y) \bar{J} Z\} .
\end{aligned}
$$

A submanifold $(M, g)$ of a semi-Riemannian manifold $(\bar{M}, \bar{g})$ of codimension 2 is called a half-lightlike submanifold if the radical distribution $\operatorname{Rad}(T M)=T M \cap T M^{\perp}$ is a vector subbundle of the tangent bundle TM and the normal bundle $T M^{\perp}$ is of rank 1 , where the metric $g$ induced from ambient space $\bar{M}$ is degenerate. Thus there exist nondegenerate complementary distributions $S(T M)$ and $S\left(T M^{\perp}\right)$ of $\operatorname{Rad}(T M)$ in $T M$ and $T M^{\perp}$ respectively, which are called the screen and screen transversal distributions on $M$, respectively. Thus we have

$$
\begin{aligned}
T M & =\operatorname{Rad}(T M) \oplus_{\text {orth }} S(T M), \\
T M^{\perp} & =\operatorname{Rad}(T M) \oplus_{\text {orth }} S\left(T M^{\perp}\right),
\end{aligned}
$$

where $\oplus_{\text {orth }}$ denotes the orthogonal direct sum. Consider the orthogonal complementary distribution $S(T M)^{\perp}$ to $S(T M)$ in $T \bar{M}$; it is easy to see that $T M^{\perp}$ is a subbundle of $S(T M)^{\perp}$. As $S\left(T M^{\perp}\right)$ is a nondegenerate subbundle of $S(T M)^{\perp}$, then the orthogonal complementary distribution $S\left(T M^{\perp}\right)^{\perp}$ to $S\left(T M^{\perp}\right)$ in $S(T M)^{\perp}$ is also a nondegenerate distribution. Clearly $\operatorname{Rad}(T M)$ is a subbundle of $S\left(T M^{\perp}\right)^{\perp}$. Choose $L \epsilon$ $\Gamma\left(S\left(T M^{\perp}\right)\right)$ as a unit vector field with $\bar{g}(L, L)= \pm 1$. In this paper, we may assume that $\bar{g}(L, L)=1$ without loss of generality. For any null section $\xi \in \operatorname{Rad}(T M)$, there exists a uniquely defined null vector field $N \in \Gamma\left(S\left(T M^{\perp}\right)^{\perp}\right)$ satisfying

$$
\begin{array}{r}
\bar{g}(\xi, N)=1, \quad \bar{g}(N, N)=\bar{g}(N, X)=\bar{g}(N, L)=0, \\
\forall X \in \Gamma(S(T M)) .
\end{array}
$$

Denote by $\operatorname{ltr}(T M)$ the vector subbundle of $S\left(T M^{\perp}\right)^{\perp}$ locally spanned by $N$. Then we show that $S\left(T M^{\perp}\right)^{\perp}=\operatorname{Rad}(T M) \oplus$ $\operatorname{ltr}(T M)$. We put $\operatorname{tr}(T M)=S\left(T M^{\perp}\right) \oplus_{\text {orth }} \operatorname{ltr}(T M)$. We call
$N, \operatorname{ltr}(T M)$, and $\operatorname{tr}(T M)$ the lightlike transversal vector field, lightlike transversal vector bundle, and transversal vector bundle of $M$ with respect to the chosen screen distribution $S(T M)$, respectively. Then $T \bar{M}$ is decomposed as follows:

$$
\begin{aligned}
T \bar{M}= & T M \oplus \operatorname{tr}(T M) \\
= & \{\operatorname{Rad}(T M) \oplus \operatorname{tr}(T M)\} \oplus_{\text {orth }} S(T M) \\
= & \{\operatorname{Rad}(T M) \oplus \operatorname{ltr}(T M)\} \\
& \oplus_{\text {orth }} S(T M) \oplus_{\text {orth }} S\left(T M^{\perp}\right) .
\end{aligned}
$$

Let $P$ be the projection morphism of $T M$ on $S(T M)$ with respect to the decomposition (7). For any $X, Y \in \Gamma(T M), N \in$ $\Gamma(\operatorname{ltr}(T M)), \xi \in \Gamma(\operatorname{Rad}(T M))$, and $L \in \Gamma\left(S(T M)^{\perp}\right)$, the Gauss and Weingarten formulas of $M$ and $S(T M)$ are given by

$$
\begin{gathered}
\bar{\nabla}_{X} Y=\nabla_{X} Y+B(X, Y) N+D(X, Y) L, \\
\bar{\nabla}_{X} N=-A_{N} X+\tau(X) N+\rho(X) L, \\
\bar{\nabla}_{X} L=-A_{L} X+\phi(X) N, \\
\nabla_{X} P Y=\nabla_{X}^{*} P Y+C(X, P Y) \xi, \\
\nabla_{X} \xi=-A_{\xi}^{*} X-\tau(X) \xi,
\end{gathered}
$$

where $\nabla$ and $\nabla^{*}$ are induced connection on $T M$ and $S(T M)$, respectively; $B$ and $D$ are called locally second fundamental forms of $M$; and $C$ is called the locally second fundamental form on $S(T M) . A_{N}, A_{\xi}^{*}$, and $A_{L}$ are linear operators on $T M$ and $\tau, \rho$, and $\phi$ are 1 -forms on $T M$. We put $h(X, Y)=$ $B(X, Y) N+D(X, Y) L$ for any $X, Y \in \Gamma(T M)$, where $h$ is called the second fundamental form of $M$. Note that the connection $\nabla$ is torsion free but is not metric tensor and the connection $\nabla^{*}$ is metric. We also know that both $B$ and $D$ are symmetric tensors on $\Gamma(T M)$ and independent of the choice of a screen distribution. Using (8)-(12) we obtain the following equations:

$$
\begin{gathered}
B(X, \xi)=0, \quad D(X, \xi)=-\phi(X), \\
\bar{\nabla}_{X} \xi=-A_{\xi}^{*} X-\tau(X) \xi-\phi(X) L \\
g\left(A_{N} X, P Y\right)=\bar{g}\left(N, h^{*}(X, P Y)\right), \quad \bar{g}\left(A_{N} X, N\right)=0 \\
g\left(A_{\xi}^{*} X, P Y\right)=\bar{g}(\xi, h(X, P Y)), \quad \bar{g}\left(A_{\xi}^{*} X, N\right)=0 \\
\left(\nabla_{X} g\right)(Y, Z)=B(X, Y) \eta(Z)+B(X, Z) \eta(Y),
\end{gathered}
$$

for any $X, Y, Z \in \Gamma(T M)$, where $\eta(X)=\bar{g}(X, N)$. Denote by $\bar{R}$ and $R$ the curvature tensor of semi-Riemannian connection $\bar{\nabla}$ of $\bar{M}$; then we have

$$
\begin{aligned}
& \bar{R}(X, Y) Z=R(X, Y) Z+B(X, Z) A_{N} Y-B(Y, Z) A_{N} X \\
&+ D(X, Z) A_{L} Y-D(Y, Z) A_{L} X \\
&+\left\{\left(\nabla_{X} B\right)(Y, Z)-\left(\nabla_{Y} B\right)(X, Z)\right. \\
&+\tau(X) B(Y, Z)-\tau(Y) B(X, Z) \\
&+\phi(X) D(Y, Z)-\phi(Y) D(X, Z)\} N \\
&+\left\{\left(\nabla_{X} D\right)(Y, Z)-\left(\nabla_{Y} D\right)(X, Z)\right. \\
&+\rho(X) B(Y, Z)-\rho(Y) B(X, Z)\} L .
\end{aligned}
$$




\section{Semiparallel Half-Lightlike Submanifolds}

In this section, we mainly investigate semiparallel half-lightlike submanifolds of indefinite Kenmotsu manifolds. First of all, we need the following two lemmas.

Lemma 1 (see [7]). Let $M$ be a half-lightlike submanifold of an indefinite almost contact metric manifolds $\bar{M}$. Then there exists a screen distribution $S(T M)$ such that

$$
\bar{J}\left(S(T M)^{\perp}\right) \subset S(T M) .
$$

Using the similar method shown in [15], it is easy to see the following result.

Lemma 2. Let $M$ be a half-lightlike submanifold of an indefinite Kenmotsu manifolds $\bar{M}$. Then the structure vector field $\zeta$ does not belong to $\operatorname{Rad}(T M)$ and $\operatorname{ltr}(T M)$.

If the structure vector field $\zeta$ is tangent to $M$, then $\zeta$ belongs to $\Gamma(S(T M))$. We say that a half-lightlike submanifold $M$ of an indefinite Kenmotsu manifold $\bar{M}$ is tangential if $M$ is tangent to the characteristic vector field $\zeta$ of $\bar{M}$. Thus, by using the above two lemmas we see that there exists a nondegenerate almost complex distribution $D_{0}$ on $M$ with respect to $\bar{J}$; that is, $\bar{J}\left(D_{0}\right)=D_{0}$, such that

$$
\begin{aligned}
S(T M)= & \{\bar{J}(\operatorname{Rad}(T M)) \oplus \bar{J}(\operatorname{ltr}(T M))\} \\
& \oplus_{\text {orth }} \bar{J}\left(S\left(T M^{\perp}\right)\right) \oplus_{\text {orth }} D_{0} .
\end{aligned}
$$

Thus, the general decompositions (5) and (7) reduce the following:

$$
\begin{gathered}
T M=D \oplus D^{\prime}, \quad T \bar{M}=D \oplus D^{\prime} \oplus_{\text {orth }} \operatorname{tr}(T M), \\
D=\bar{J}(\operatorname{Rad}(T M)) \oplus_{\text {orth }} \operatorname{Rad}(T M) \oplus_{\text {orth }} D_{0}, \\
D^{\prime}=\bar{J}(\operatorname{ltr}(T M)) \oplus_{\text {orth }} \bar{J}\left(S\left(T M^{\perp}\right)\right),
\end{gathered}
$$

where $D$ is an almost complex distribution of $M$ with respect to $\bar{J}$. Consider a pair of local null vectors $\{U, V\}$ and a local nonnull vector field $W$ on $S(T M)$ defined by

$$
U=-\bar{J} N, \quad V=-\bar{J} \xi, \quad W=-\bar{J} L .
$$

Denote by $\delta$ the projection morphism of TM on $D$ with respect to the decomposition (20); then for any vector field $X$ on $M$ we have

$$
\begin{aligned}
& X=\mathcal{S} X+u(X) U+\omega(X) W, \\
& \bar{J} X=J X+u(X) N+\omega(X) L,
\end{aligned}
$$

where $u, v$, and $\omega$ are 1-forms locally defined on $M$ by

$$
u(X)=g(X, V), \quad v(X)=g(X, U), \quad \omega(X)=g(X, W)
$$

and $J$ is a tensor of type $(1,1)$ globally defined on $M$ by $J=\bar{J}$ 。 $\mathcal{S}$. From the second term of (3) and (8) we have the following identities:

$$
\nabla_{X} \zeta=-X+\theta(X) \zeta, \quad B(X, \zeta)=0, \quad D(X, \zeta)=0
$$

for any $X \in \Gamma(T M)$.
Definition 3. Let $(M, g, S(T M))$ be a half-lightlike submanifold of an indefinite Kenmotsu manifold $(\bar{M}, \bar{J}, \zeta, \theta, \bar{g})$, tangent to the characteristic vector $\zeta$. Then $M$ is said to be semiparallel if its second fundamental form $h$ satisfies

$$
\begin{aligned}
& (R(X, Y) \cdot h)\left(X_{1}, X_{2}\right) \\
& \quad=-h\left(R(X, Y) X_{1}, X_{2}\right)-h\left(X_{1}, R(X, Y) X_{2}\right)=0
\end{aligned}
$$

for any $X, Y, X_{1}, X_{2} \in \Gamma(T M)$.

Definition 4 (see [1]). A half-lightlike submanifold $M$ of a semi-Riemannian manifold $\bar{M}$ is said to be irrotational if $\bar{\nabla}_{X} \xi \in \Gamma(T M)$ for any $X \in \Gamma(T M)$.

From (8) and (12) we see that a necessary and sufficient condition for a half-lightlike submanifold $M$ to be irrotational is $D(X, \xi)=\phi(X)=0$ for any $X \in \Gamma(T M)$.

Lemma 5. Let $(M, g, S(T M))$ be a half-lightlike submanifold of an indefinite Kenmotsu space from $(\bar{M}(c), \bar{g})$; then the curvature tensor of $M$ is given by

$$
\begin{aligned}
& R(X, Y) Z \\
& =\frac{c-3}{4}\{\bar{g}(Y, Z) X-\bar{g}(X, Z) Y\} \\
& +\frac{c+1}{4}\{\theta(X) \theta(Z) Y-\theta(Y) \theta(Z) X \\
& +\bar{g}(X, Z) \theta(Y) \zeta-\bar{g}(Y, Z) \theta(X) \zeta \\
& +\bar{g}(\bar{J} Y, Z) J X-\bar{g}(\bar{J} X, Z) J Y \\
& -2 \bar{g}(\bar{J} X, Y) J Z\} \\
& \text { - } B(X, Z) A_{N} Y+B(Y, Z) A_{N} X \\
& \text { - } D(X, Z) A_{L} Y+D(Y, Z) A_{L} X
\end{aligned}
$$

for any $X, Y, Z \in \Gamma(T M)$.

Proof. The proof follows from (4) and (17).

Theorem 6. Let (M, g, S(TM)) be a semiparallel half-lightlike submanifold of an indefinite Kenmotsu space from $(\bar{M}(c), \bar{g})$, tangent to the characteristic vector $\zeta$. If $M$ is irrotational, then either $c=-1$ or $M$ is $(\bar{J}(\operatorname{Rad}(T M)), T M)$-mixed geodesic.

Proof. Putting (27) and $h(X, Y)=B(X, Y) N+D(X, Y) L$ into (26), and using the decomposition (7) and (25) we obtain

$$
\begin{aligned}
0= & \frac{c-3}{4} \\
\times & \left\{\bar{g}\left(Y, X_{1}\right) B\left(X, X_{2}\right)-\bar{g}\left(X, X_{1}\right) B\left(Y, X_{2}\right)\right. \\
& \left.+\bar{g}\left(Y, X_{2}\right) B\left(X, X_{1}\right)-\bar{g}\left(X, X_{2}\right) B\left(Y, X_{1}\right)\right\}
\end{aligned}
$$




$$
\begin{aligned}
& +\frac{c+1}{4}\left\{\theta(X) \theta\left(X_{1}\right) B\left(Y, X_{2}\right)-\theta(Y) \theta\left(X_{1}\right) B\left(X, X_{2}\right)\right. \\
& +\theta(X) \theta\left(X_{2}\right) B\left(Y, X_{1}\right)-\theta(Y) \theta\left(X_{2}\right) B\left(X, X_{1}\right) \\
& +\bar{g}\left(\bar{J} Y, X_{1}\right) B\left(J X, X_{2}\right)-\bar{g}\left(\bar{J} X, X_{1}\right) B\left(J Y, X_{2}\right) \\
& +\bar{g}\left(\bar{J} Y, X_{2}\right) B\left(J X, X_{1}\right)-\bar{g}\left(\bar{J} X, X_{2}\right) B\left(J Y, X_{1}\right) \\
& \left.-2 \bar{g}(\bar{J} X, Y) B\left(J X_{1}, X_{2}\right)-2 \bar{g}(\bar{J} X, Y) B\left(J X_{2}, X_{1}\right)\right\} \\
& -B\left(X, X_{1}\right) B\left(A_{N} Y, X_{2}\right)+B\left(Y, X_{1}\right) B\left(A_{N} X, X_{2}\right) \\
& -B\left(X, X_{2}\right) B\left(A_{N} Y, X_{1}\right)+B\left(Y, X_{2}\right) B\left(A_{N} X, X_{1}\right) \\
& -D\left(X, X_{1}\right) B\left(A_{L} Y, X_{2}\right)+D\left(Y, X_{1}\right) B\left(A_{L} X, X_{2}\right) \\
& -D\left(X, X_{2}\right) B\left(A_{L} Y, X_{1}\right)+D\left(Y, X_{2}\right) B\left(A_{L} X, X_{1}\right), \\
& 0=\frac{c-3}{4} \\
& \times\left\{\bar{g}\left(Y, X_{1}\right) D\left(X, X_{2}\right)-\bar{g}\left(X, X_{1}\right) D\left(Y, X_{2}\right)\right. \\
& \left.+\bar{g}\left(Y, X_{2}\right) D\left(X, X_{1}\right)-\bar{g}\left(X, X_{2}\right) D\left(Y, X_{1}\right)\right\} \\
& +\frac{c+1}{4}\left\{\theta(X) \theta\left(X_{1}\right) D\left(Y, X_{2}\right)-\theta(Y) \theta\left(X_{1}\right) D\left(X, X_{2}\right)\right. \\
& +\theta(X) \theta\left(X_{2}\right) D\left(Y, X_{1}\right)-\theta(Y) \theta\left(X_{2}\right) D\left(X, X_{1}\right) \\
& +\bar{g}\left(\bar{J} Y, X_{1}\right) D\left(J X, X_{2}\right)-\bar{g}\left(\bar{J} X, X_{1}\right) D\left(J Y, X_{2}\right) \\
& +\bar{g}\left(\bar{J} Y, X_{2}\right) D\left(J X, X_{1}\right)-\bar{g}\left(\bar{J} X, X_{2}\right) D\left(J Y, X_{1}\right) \\
& \left.-2 \bar{g}(\bar{J} X, Y) D\left(J X_{1}, X_{2}\right)-2 \bar{g}(\bar{J} X, Y) D\left(J X_{2}, X_{1}\right)\right\} \\
& -B\left(X, X_{1}\right) D\left(A_{N} Y, X_{2}\right)+B\left(Y, X_{1}\right) D\left(A_{N} X, X_{2}\right) \\
& -B\left(X, X_{2}\right) D\left(A_{N} Y, X_{1}\right)+B\left(Y, X_{2}\right) D\left(A_{N} X, X_{1}\right) \\
& \text { - } D\left(X, X_{1}\right) D\left(A_{L} Y, X_{2}\right)+D\left(Y, X_{1}\right) D\left(A_{L} X, X_{2}\right) \\
& -D\left(X, X_{2}\right) D\left(A_{L} Y, X_{1}\right)+D\left(Y, X_{2}\right) D\left(A_{L} X, X_{1}\right) \text {. }
\end{aligned}
$$

Replacing $X$ by $\xi$ in (28) and (29), respectively, and noting that $M$ is irrotational, then we obtain

$$
\begin{aligned}
0= & \frac{c+1}{4} \\
& \times\left\{\bar{g}\left(\bar{J} Y, X_{1}\right) B\left(J \xi, X_{2}\right)+u\left(X_{1}\right) B\left(J Y, X_{2}\right)\right. \\
& +\bar{g}\left(\bar{J} Y, X_{2}\right) B\left(J \xi, X_{1}\right)+u\left(X_{2}\right) B\left(J Y, X_{1}\right) \\
& \left.+2 u(Y) B\left(J X_{1}, X_{2}\right)+2 u(Y) B\left(J X_{2}, X_{1}\right)\right\} \\
& +B\left(Y, X_{1}\right) B\left(A_{N} \xi, X_{2}\right)+B\left(Y, X_{2}\right) B\left(A_{N} \xi, X_{1}\right) \\
& +D\left(Y, X_{1}\right) B\left(A_{L} \xi, X_{2}\right)+D\left(Y, X_{2}\right) B\left(A_{L} \xi, X_{1}\right) \\
0 & \frac{c+1}{4} \quad \\
& \times\left\{\bar{g}\left(\bar{J} Y, X_{1}\right) D\left(J \xi, X_{2}\right)+u\left(X_{1}\right) D\left(J Y, X_{2}\right)\right. \\
& +\bar{g}\left(\bar{J} Y, X_{2}\right) D\left(J \xi, X_{1}\right)+u\left(X_{2}\right) D\left(J Y, X_{1}\right)
\end{aligned}
$$

$$
\begin{array}{r}
\left.+2 u(Y) D\left(J X_{1}, X_{2}\right)+2 u(Y) D\left(J X_{2}, X_{1}\right)\right\} \\
+B\left(Y, X_{1}\right) D\left(A_{N} \xi, X_{2}\right)+B\left(Y, X_{2}\right) D\left(A_{N} \xi, X_{1}\right) \\
+D\left(Y, X_{1}\right) D\left(A_{L} \xi, X_{2}\right)+D\left(Y, X_{2}\right) D\left(A_{L} \xi, X_{1}\right) .
\end{array}
$$

Substituting $X_{2}=\xi$ into (30) and (31), respectively, then we get

$$
\begin{aligned}
& \frac{c+1}{4} u(Y) B\left(V, X_{1}\right)=0, \\
& \frac{c+1}{4} u(Y) D\left(V, X_{1}\right)=0 .
\end{aligned}
$$

Finally, substituting $Y=U$ into (32) and (33) implies that $(c+1) B\left(V, X_{1}\right)=(c+1) D\left(V, X_{1}\right)=0$ for any $X_{1} \in \Gamma(T M)$. Thus, it follows that either $c=-1$ or $B\left(V, X_{1}\right)=D\left(V, X_{1}\right)=0$ for any $X_{1} \in \Gamma(T M)$, which completes the proof.

\section{Parallel Half-Lightlike Submanifolds}

In this section, we mainly prove some properties of parallel half-lightlike submanifolds of indefinite Kenmotsu manifolds.

Lemma 7. Let $(M, g, S(T M))$ be a half-lightlike submanifold of an indefinite Kenmotsu space from $(\bar{M}(c), \bar{g})$; then the local second fundamental forms $B$ and $D$ are given, respectively, by

$$
\begin{aligned}
\frac{c+1}{4}\{\bar{g}(\bar{J} Y, Z) u(X)-\bar{g}(\bar{J} X, Z) u(Y)-2 \bar{g}(\bar{J} X, Y) u(Z)\} \\
=\left(\nabla_{X} B\right)(Y, Z)-\left(\nabla_{Y} B\right)(X, Z)+\tau(X) B(Y, Z) \\
\quad-\tau(Y) B(X, Z)+\phi(X) D(Y, Z)-\phi(Y) D(X, Z),
\end{aligned}
$$

$$
\begin{aligned}
\frac{c+1}{4}\{ & \bar{g}(\bar{J} Y, Z) \omega(X)-\bar{g}(\bar{J} X, Z) \omega(Y)-2 \bar{g}(\bar{J} X, Y) \omega(Z)\} \\
= & \left(\nabla_{X} D\right)(Y, Z)-\left(\nabla_{Y} D\right)(X, Z)+\rho(X) B(Y, Z) \\
& -\rho(Y) B(X, Z)
\end{aligned}
$$

for any $X, Y, Z \in \Gamma(T M)$.

Proof. The proof follows from (4) and (17).

Definition 8 . Let $(M, g, S(T M))$ be a half-lightlike submanifold of an indefinite Kenmotsu manifold $(\bar{M}, \bar{J}, \zeta, \theta, \bar{g})$, tangent to the characteristic vector $\zeta$. Then $M$ is said to be parallel (see [12]) with respect to $\nabla$ if its second fundamental form $h$ satisfies

$$
\left(\nabla_{X} h\right)(Y, Z)=0
$$

for any $X, Y, Z \in \Gamma(T M)$.

Using $h(X, Y)=B(X, Y) N+D(X, Y) L$, then a straightforward calculation gives that $M$ is said to be with the parallel second fundamental form $h$ if and only if

$$
\begin{gathered}
\left(\nabla_{X} B\right)+\tau(X) B+\phi(X) D=0, \\
\left(\nabla_{X} D\right)+\rho(X) B=0
\end{gathered}
$$

for any $X \in \Gamma(T M)$. 
Theorem 9. Let $(M, g, S(T M))$ be a parallel half-lightlike submanifold of an indefinite Kenmotsu space from $(\bar{M}(c), \bar{g})$, tangent to the characteristic vector $\zeta$. Then $c=-1$ and hence $M$ is totally geodesic.

Proof. Substituting the second term of (37) into (35) gives

$$
\begin{aligned}
\frac{c+1}{4} & \{\bar{g}(\bar{J} Y, Z) \omega(X)-\bar{g}(\bar{J} X, Z) \omega(Y)-2 \bar{g}(\bar{J} X, Y) \omega(Z)\} \\
& =0
\end{aligned}
$$

for any $X, Y, Z \in \Gamma(T M)$. Replacing $Y$ and $Z$ by $\xi$ and $U$, respectively, in (38), we obtain

$$
\frac{c+1}{4} \omega(X)=0, \quad \forall X \in \Gamma(T M) .
$$

Thus, substituting $X=W$ into (39) gives $c=-1$. From (25) and (37) we have

$$
\begin{gathered}
\left(\nabla_{X} B\right)(Y, \zeta)=-\tau(X) B(Y, \zeta)-\phi(X) D(Y, \zeta)=0, \\
\left(\nabla_{X} D\right)(Y, \zeta)=-\rho(X) B(Y, \zeta)=0
\end{gathered}
$$

for any $X, Y \in \Gamma(T M)$. On the other hand, it follows from the first term of (25) and (40) that

$$
\begin{aligned}
\left(\nabla_{X} B\right)(Y, \zeta) & =X(B(Y, \zeta))-B\left(\nabla_{X} Y, \zeta\right)-B\left(Y, \nabla_{X} \zeta\right) \\
& =B(X, Y)=0 \\
\left(\nabla_{X} D\right)(Y, \zeta) & =X(D(Y, \zeta))-D\left(\nabla_{X} Y, \zeta\right)-D\left(Y, \nabla_{X} \zeta\right) \\
& =D(X, Y)=0
\end{aligned}
$$

for any $X, Y \in \Gamma(T M)$. Thus, the proof follows from (41).

Let $(M, g, S(T M))$ be a half-lightlike submanifold of an indefinite Kenmotsu manifold. We say that the local second fundamental forms of $M$ are parallel if and only if $\nabla_{X} B=0$ and $\nabla_{X} D=0$ for any $X \in \Gamma(T M)$.

Lemma 10. Let $(M, g, S(T M))$ be a half-lightlike submanifold of an indefinite Kenmotsu space from $(\bar{M}(c), \bar{g})$, tangent to the characteristic vector $\zeta$. If the local second fundamental form $D$ is parallel with respect to $\nabla$, then $c=-1$. Moreover, in this case either $\rho(\xi)=0$ or $B=0$.

Proof. Suppose that the local second fundamental form $D$ is parallel with respect to $\nabla$; that is, $\nabla_{X} D=0$ for any $X \in$ $\Gamma(T M)$. Then it follows from (35) that

$$
\begin{aligned}
\frac{c+1}{4} & \{\bar{g}(\bar{J} Y, Z) \omega(X)-\bar{g}(\bar{J} X, Z) \omega(Y)-2 \bar{g}(\bar{J} X, Y) \omega(Z)\} \\
& =\rho(X) B(Y, Z)-\rho(Y) B(X, Z)
\end{aligned}
$$

for any $X, Y, Z \in \Gamma(T M)$. Substituting $Z=\xi$ into (42) and using $B(X, \xi)=0$ and $\bar{g}(\xi, W)=0$, we obtain

$$
\frac{c+1}{4}\{\bar{g}(\bar{J} Y, \xi) \omega(X)-\bar{g}(\bar{J} X, \xi) \omega(Y)\}=0
$$

for any $X, Y \in \Gamma(T M)$. Putting $Y=U$ into (43) and using (1) give that $((c+1) / 4) \omega(X)=0$ for any $X \in \Gamma(T M)$; finally, replacing $X$ by $W$ in this equation gives $c=-1$.

Using $c=-1$ in (35) and noting that $D$ is parallel with respect to $\nabla$, then we have

$$
\rho(X) B(Y, Z)-\rho(Y) B(X, Z)=0
$$

for any $X, Y, Z \in \Gamma(T M)$. Replacing $X$ by $\xi$ in (44) and using $B(X, \xi)=0$ give $\rho(\xi) B(Y, Z)=0$ for $Y, Z \in \Gamma(T M)$, which completes the proof.

Theorem 11. Let $(M, g, S(T M))$ be a half-lightlike submanifold of an indefinite Kenmotsu space from $(\bar{M}(c), \bar{g})$, tangent to the characteristic vector $\zeta$. If the local second fundamental forms $B$ and $D$ are parallel with respective to $\nabla$, then $c=-1$. Moreover, if $\rho(\xi) \neq 0$ and $\phi(\xi) \neq 0$, then $M$ is $S(T M)$-totally geodesic if and only if $\bar{\nabla}_{X} \xi \in \Gamma(T M)$ for any $X \in \Gamma(S(T M))$.

Proof. Using the parallelism of two local second fundamental forms $B$ and $D$ and Lemma 10 , it follows from (34) that

$$
\begin{aligned}
\tau(X) & B(Y, Z)-\tau(Y) B(X, Z)+\phi(X) D(Y, Z) \\
- & \phi(Y) D(X, Z) \\
& =0
\end{aligned}
$$

for any $X, Y, Z \in \Gamma(T M)$. If $\rho(\xi) \neq 0$, then from Lemma 10 we know $B=0$; thus

$$
\phi(X) D(Y, Z)-\phi(Y) D(X, Z)=0
$$

for any $X, Y, Z \in \Gamma(T M)$. Replacing $X$ by $\xi$ in (46) and using (13), we obtain

$$
\phi(\xi) D(Y, Z)=-\phi(Y) \phi(Z)
$$

for any $Y, Z \in \Gamma(T M)$. Then the proof follows from (47) and Lemma 10

\section{Acknowledgments}

This work is supported by NSFC (no. 10931005) and Natural Science Foundation of Guangdong Province of China (no. S2011010000471).

\section{References}

[1] D. N. Kupeli, Singular Semi-Riemannian Geometry, vol. 366 of Mathematics and Its Applications, Kluwer Academic Publishers, Dordrecht, The Netherlands, 1996.

[2] K. L. Duggal and A. Bejancu, Lightlike submanifolds of semiRiemannian manifolds and applications, vol. 364 of Mathematics and Its Applications, Kluwer Academic Publishers, Dordrecht, The Netherlands, 1996.

[3] K. L. Duggal and D. H. Jin, Null Curves and Hypersurfaces of Semi-Riemannian Manifolds, World Scientific Publishing, Hackensack, NJ, USA, 2007.

[4] K. L. Duggal and B. Sahin, Differential Geometry of Lightlike Submanifolds, Frontiers in Mathematics, Birkhäuser, Basel, Switzerland, 2010. 
[5] K. L. Duggal and A. Bejancu, "Lightlike submanifolds of codimension two," Mathematics Journal of Toyama University, vol. 15, pp. 59-82, 1992.

[6] K. L. Duggal and D. H. Jin, "Half lightlike submanifolds of codimension 2," Mathematics Journal of Toyama University, vol. 22, pp. 121-161, 1999.

[7] D. H. Jin, "Half lightlike submanifolds of an indefinite Sasakian manifold," Journal of the Korea Society of Mathematical Education B, vol. 18, no. 2, pp. 173-183, 2011.

[8] D. Ferus, "Immersions with parallel second fundamental form," Mathematische Zeitschrift, vol. 140, pp. 87-93, 1974.

[9] J. Deprez, "Semiparallel surfaces in Euclidean space," Journal of Geometry, vol. 25, no. 2, pp. 192-200, 1985.

[10] F. Massamba, "On lightlike geometry in indefinite Kenmotsu manifolds," Mathematica Slovaca, vol. 62, no. 2, pp. 315-344, 2012.

[11] F. Massamba, "Semi-parallel lightlike hypersurfaces of indefinite Sasakian manifolds," International Journal of Contemporary Mathematical Sciences, vol. 3, no. 13-16, pp. 629-634, 2008.

[12] F. Massamba, "Lightlike hypersurfaces of indefinite Sasakian manifolds with parallel symmetric bilinear forms," Differential Geometry-Dynamical Systems, vol. 10, pp. 226-234, 2008.

[13] F. Massamba, "On semi-parallel lightlike hypersurfaces of indefinite Kenmotsu manifolds," Journal of Geometry, vol. 95, no. 1-2, pp. 73-89, 2009.

[14] A. Upadhyay and R. S. Gupta, "Semi-parallel lightlike hypersurfaces of indefinite cosymplectic space forms," Institut Mathématique. Nouvelle Série, vol. 89, no. 103, pp. 69-75, 2011.

[15] D. H. Jin, "Special half lightlike submanifolds of an indefinite cosymplectic manifold," Journal of Function Spaces and Applications, vol. 2012, Article ID 636242, 16 pages, 2012. 


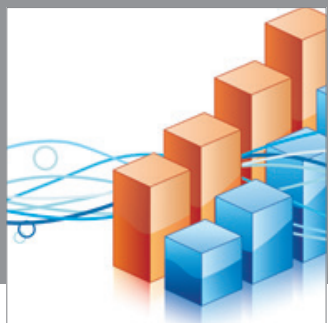

Advances in

Operations Research

mansans

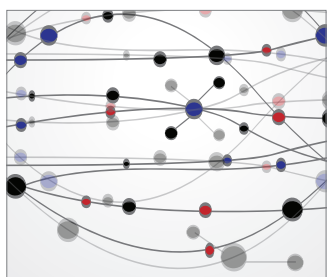

The Scientific World Journal
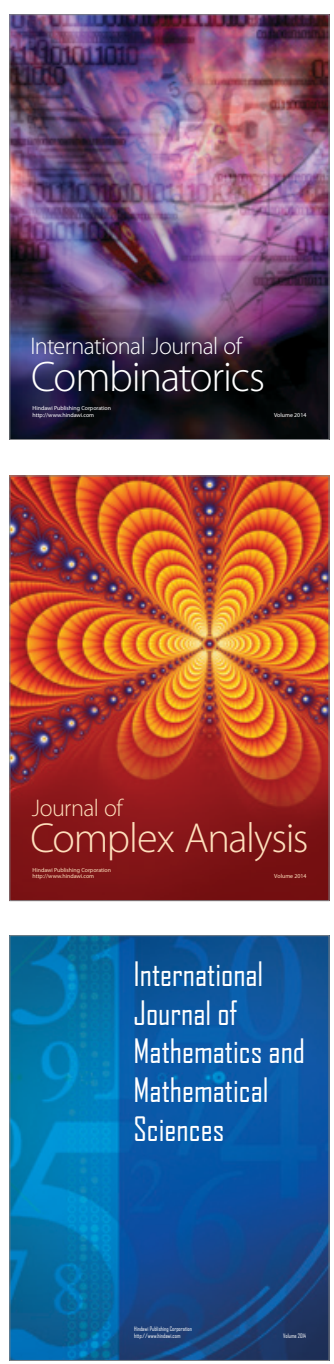
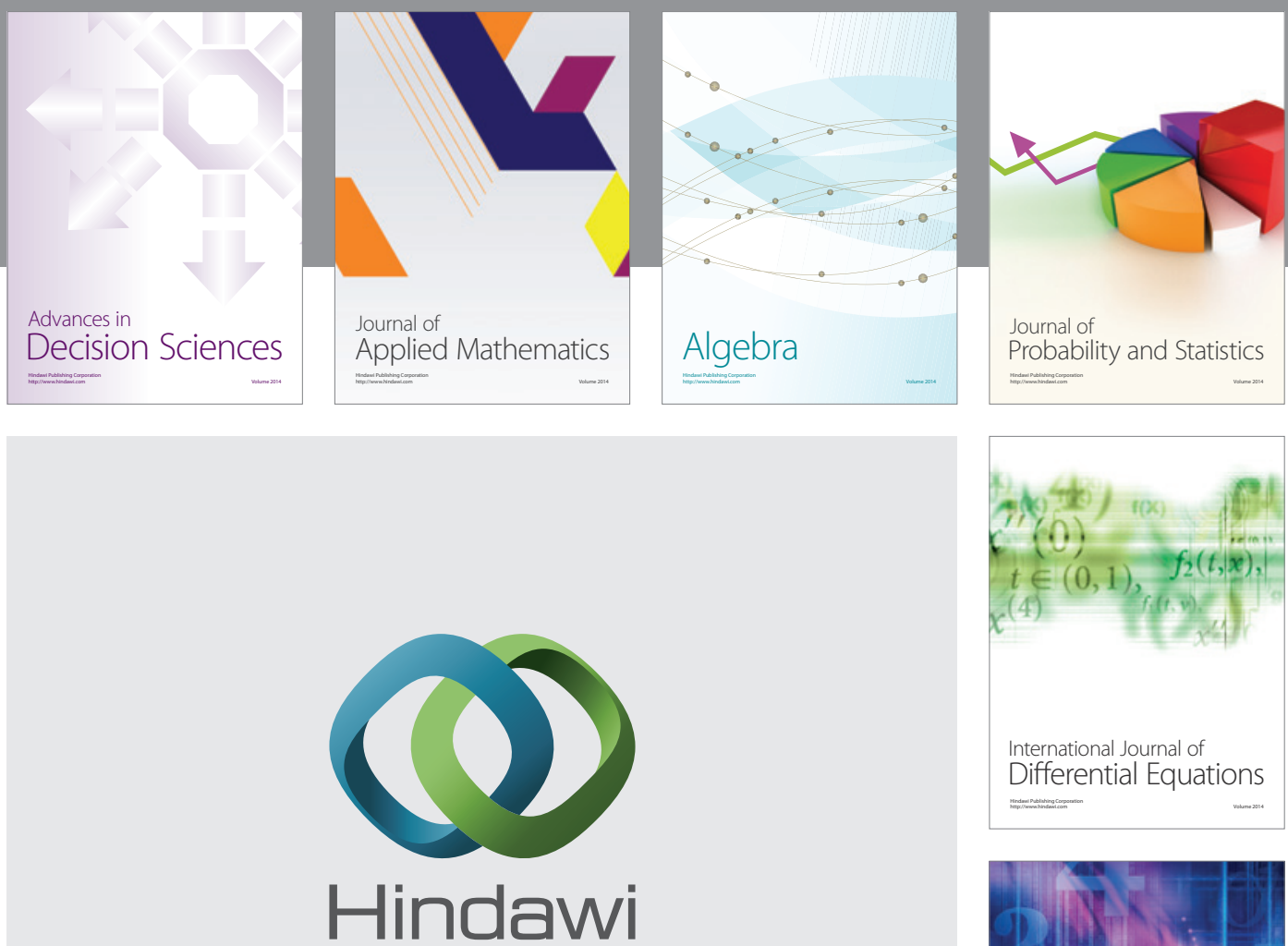

Submit your manuscripts at http://www.hindawi.com
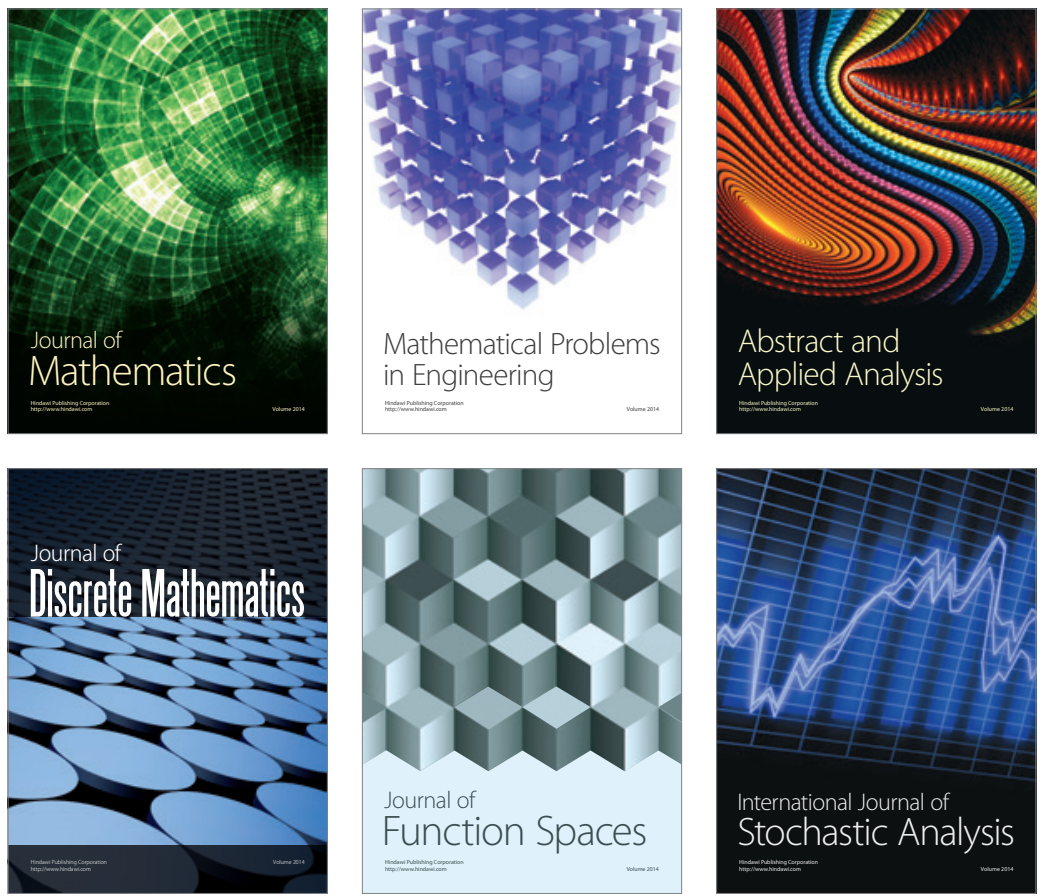

Journal of

Function Spaces

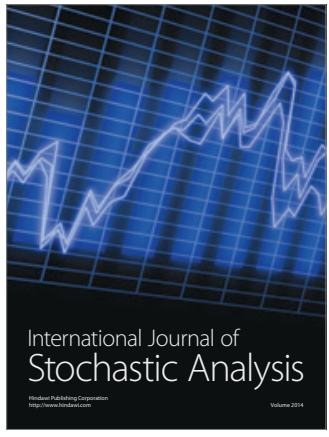

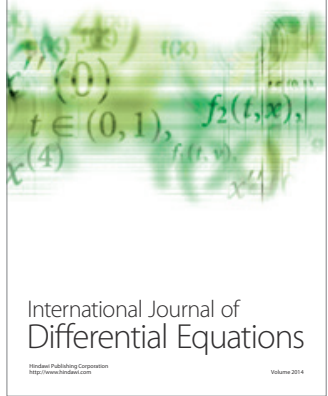
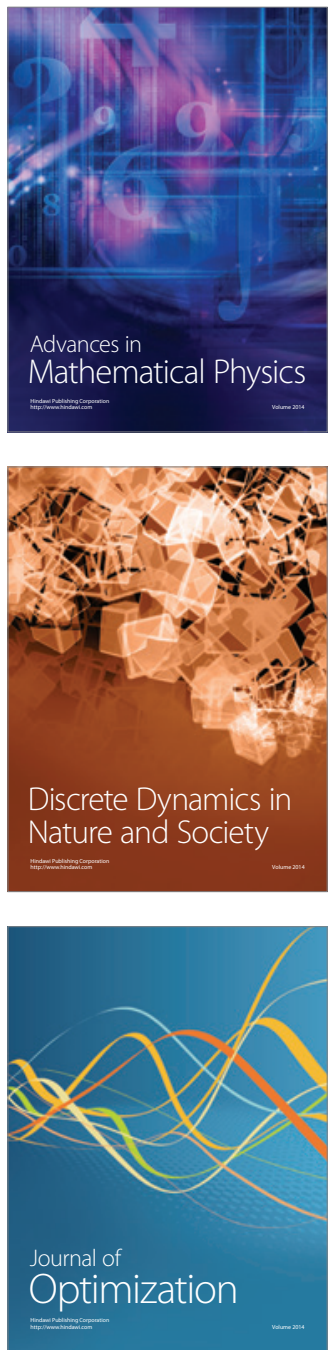